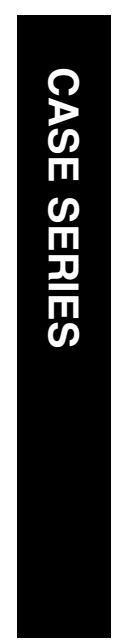

\section{Surgically induced necrotising scleritis following three-port pars plana vitrectomy without scleral buckling: a series of three cases}

\begin{abstract}
Aim To document the presentation, management, and prognosis of three cases of surgically induced necrotising scleritis (SINS) following three-port-pars plana vitrectomy (PPV) without scleral buckling. To discuss potential pathogeneses and treatments. Methods An interventional case series of three patients presenting to a tertiary referral unit over a 6-month period.

Results All cases were male, presenting within 6 weeks of PPV, with scleral necrosis around a sclerotomy site. Wound microbiology and systemic vasculitis screens were negative. Two cases had underlying systemic disease and had undergone previous ocular surgery. The necrosis resolved in all with aggressive immunosuppression.

Conclusions SINS following PPV is a rare occurrence requiring prompt and aggressive
\end{abstract}

Uveitis Service, Moorfields Eye Hospital, London, UK

Correspondence: AMS Morley, Uveitis Service, Moorfields Eye Hospital, 162 City Rd, London EC 1V 2PD, UK

Tel: +4420 72533411;

Fax: +442072534696.

E-mail: Susie@

morleys.net

Received: 2 August 2006 Accepted in revised form: 30 November 2006 Published online:

16 February 2007

Sponsors/grants: None Commercial interests: None Previous presentation: None

\section{Introduction}

Surgically induced necrotising scleritis (SINS) has been repeatedly described following cataract extraction, trabeculectomy, squint surgery, and scleral buckling. ${ }^{1,2}$ However, it
AMS Morley and C Pavesio

remains poorly documented following simple, unbuckled, 20-gauge three-port-pars plana vitrectomy (PPV), with only three indirect references existing. ${ }^{1,3}$ We present three new cases and discuss their pathogenesis and management. In all three, infective necrosis was excluded by wound swabs cultured on infusion broths and agar plates (blood, chocolate, and Sabouraud's).

\section{Case reports}

Case 1

A 63-year-old man underwent a routine threeport-PPV/gas for a right retinal detachment. He described previous right anterior uveitis but was systemically well. One month later he presented with severe right eye pain and necrosis around the inferotemporal sclerotomy site. An autoimmune/inflammatory screen was unremarkable. He was admitted and treated with pulsed IV methylprednisolone, which improved the pain, but the following week he developed a second superotemporal lesion despite continued treatment with oral prednisolone $(80 \mathrm{mg})$ and cyclophosphamide $(100 \mathrm{mg})$. Unfortunately, his right retina simultaneously re-detached necessitating PPV/ oil. His high-dose steroids were maintained until surgery and then tapered, and the cyclophosphamide was replaced with azathioprine $(150 \mathrm{mg} /$ day) after 6 months. The two areas of scleral necrosis resolved, leaving scleral thinning. No relapses have occurred.

Case 2

A 44-year-old man with a pseudophakic retinal detachment underwent a second routine three- 
port-PPV/gas for a re-detachment 3 months later. He was a smoker with known lung cancer. After 2 weeks, he developed painless scleral necrosis around the inferotemporal sclerotomy. Investigations confirmed a normal immune profile and ESR of $8 \mathrm{~mm} / \mathrm{h}$. Residual scleral polyglactin sutures were removed and he was treated with prednisolone $80 \mathrm{mg}$ for 3 weeks before tapering. The necrotic area progressively resolved (Figure 1). He is now off therapy and has shown no relapse.

\section{Case 3}

A 74-year-old man with proliferative diabetic retinopathy, previously treated with cryotherapy and pan-retinal photocoagulation, underwent a routine left three-port-PPV/cataract extraction/delamination/sulcus IOL/laser for a non-clearing vitreous haemorrhage. After 6 weeks, he developed severe pain with scleral necrosis around the superotemporal sclerotomy site. Immune markers and a vasculitis screen were unremarkable. He was started on prednisolone $(80 \mathrm{mg})$, cyclophosphamide (100 mg), topical steroids and antibiotics, but responded poorly and progressed to intravenous methylprednisolone the following week. However, the necrotic area continued to grow and four new areas developed (Figure 2). His lymphocyte count simultaneously dropped below $0.2 \times 10^{9} / 1$ necessitating termination of his cyclophosphamide. Owing to limited therapeutic options, he was commenced on infliximab (3 $\mathrm{mg} / \mathrm{kg} / \mathrm{month}$ ) and his steroids were reduced. After three infusions, he felt systemically unwell and was admitted for investigations and consideration of enucleation. Infliximab was discontinued but within 1 month he was comfortable and the lesions had almost resolved.

\section{Comment}

Classic SINS is thought to involve a delayed-type hypersensitivity response whereby even mild surgical trauma or ischaemia expose tissue antigens to which the immune system becomes sensitised. ${ }^{4,5}$ Alternative theories include molecular mimicry, possibly following infection, or generalised immune complex deposition from a vasculitis. ${ }^{1,4,5}$ Such hypotheses are supported by the success of systemic immunosuppressants as therapy, ${ }^{1,4,5}$ the presence of immune complexes in episcleral vessel walls ${ }^{4,5}$ and the high prevalence of vasculitis among such patients. ${ }^{1}$

Our cases showed some differences from classic SINS; ${ }^{1}$ notably an earlier postoperative presentation (4-6 weeks vs 9 months) and an absence of systemic vasculitides.

This onset brings SINS into the differential of persistent postoperative inflammation, as well as requiring careful exclusion of infective necrosis. The latter is well described following pterygium surgery, ${ }^{6,7}$ with $65-81 \%$ culturing Pseudomonas, ${ }^{6,7}$ and has also been reported following a buckled PPV. ${ }^{8}$ Although our cultures were negative, a subclinical, treated, postoperative infection still remains a possible trigger for SINS.

Bacterial superinfection can complicate the presentation further. ${ }^{9}$

Similarities to classic SINS ${ }^{1}$ included previous ocular surgery and systemic disease, present in two cases. Cases 2 and 3 had vascular risk factors that could have contributed to local ischaemia. Previous herpes zoster ophthalmicus cannot be excluded in case 1 .

The scleral polyglactin 910 sutures (Ethicon) may have also increased local inflammation and enhanced antigen presentation. Similar necrosis has been described around such sutures used in cataract surgery ${ }^{10}$ and consequently residual sutures were removed. We identified no manufacturing anomaly.

Management was as recommended for classic SINS and involved high dose systemic immunosuppression with steroids, cyclophosphamide, and azathioprine. ${ }^{1}$ Case reports have emerged describing the successful use of infliximab in non-surgical necrotising scleritis and this may also prove effective in SINS when options are limited. ${ }^{11,12}$
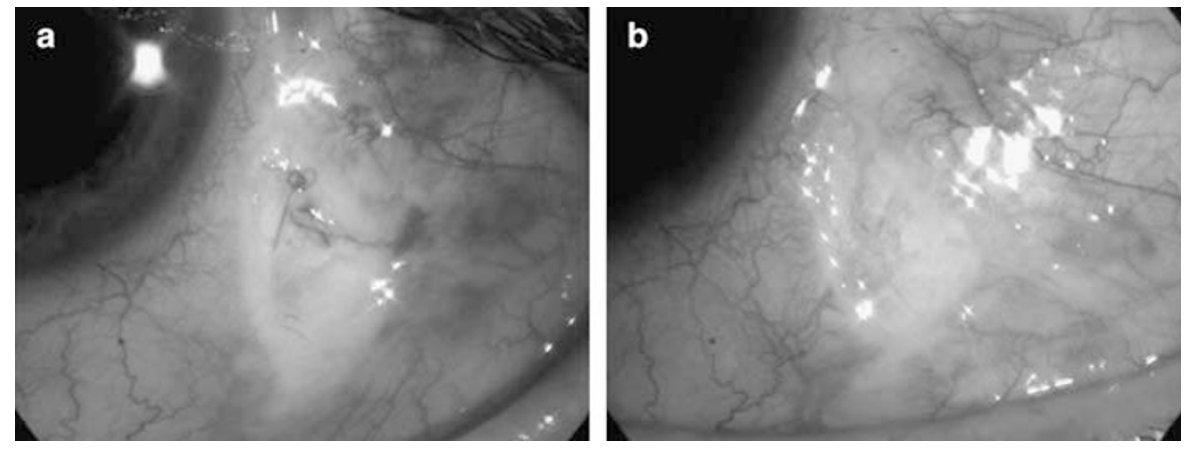

Figure 1 (a) An area of scleral necrosis is shown around the inferotemporal sclerotomy site. (b) The edges of the ulcer are no longer infiltrated, and the central area is slowly resolving. 


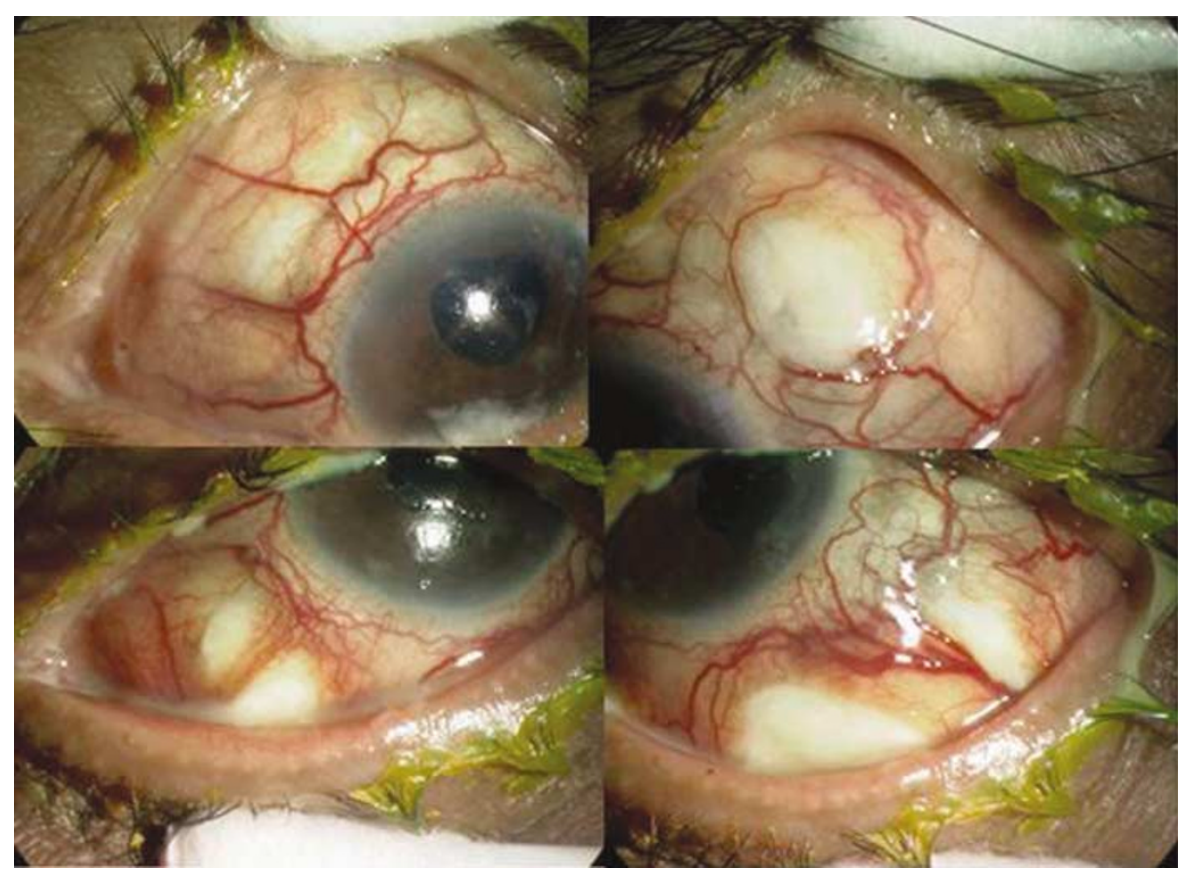

Figure 2 The original patch of scleral necrosis is seen superotemporally at the site of a sclerotomy. In addition, multiple new lesions are visible all round the anterior sclera.

SINS remains a rare but difficult complication of PPV, requiring prompt and aggressive immunosuppression. More reports of this association are required for a better understanding of its pathogenesis, treatment, and prognosis.

\section{References}

1 O'Donoghue E, Lightman S, Tuft S, Watson P. Surgically induced necrotising sclerokeratitis (SINS) - precipitating factors and response to treatment. Br J Ophthalmol 1992; 76 17-21.

2 Grindle CF, Marshall J, McLeod D, Clarke E, Fison LG. Complications of explants used in retinal detachment surgery. Mod Probl Ophthalmol 1979; 20: 219-222.

3 Kwok AK, Li KK, Lai TY, Lam DS. Pars plana vitrectomy in the management of retained intravitreal lens fragments after cataract surgery. Clin Experiment Ophthalmol 2002; 30: 399-403.

4 Diaz-Valle D, Benitez del Castillo JM, Castillo A, Sayagues O, Banares A, Garcia-Sanchez J. Immunologic and clinical evaluation of postsurgical necrotizing sclerocorneal ulceration. Cornea 1998; 17: 371-375.
5 Fong LP, Sainz de la Maza M, Rice BA, Kupferman AE, Foster CS. Immunopathology of scleritis. Ophthalmology 1991; 98: 472-479.

6 Chang-Ping L, Min-Hsiu S, Ming-Chi T. Clinical experiences of infectious scleral ulceration: a complication of pterygium operation. Br J Ophthalmol 1997; 81: 980-983.

7 Huang FC, Huang SP, Tseng SH. Management of infective scleritis after pterygium excision. Cornea 2000; 19: 34-39.

8 Zinn KM, Ferry AP. Massive scleral necrosis from a pseudomonal infection following scleral buckling and pars plana vitrectomy surgery. Mt Sinai J Med 1980; 47: 618-621.

9 Vagefi MR, Hollander DA, Seitzman GD, Margolis TP. Bilateral surgically induced necrotising scleritis with secondary superinfection. Br J Ophthalmol 2005; 89: 124-125.

10 Salamon SM, Mondino BJ, Zaidman GW. Peripheral corneal ulcers, conjunctival ulcers, and scleritis after cataract surgery. Am J Ophthalmol 1982; 93: 334-337.

11 Ashok D, Ayliffe WH, Kiely PD. Necrotizing scleritis associated with rheumatoid arthritis: long-term remission with high-dose infliximab therapy. Rheumatology (Oxford) 2005; 44: 950-951.

12 El-Shabrawi Y, Hermann J. Anti-TNF alpha therapy in chronic necrotizing scleritis resistant to standard immunomodulatory therapy in a patient with Wegener's granulomatosis. Eye 2005; 19: 1017-1018. 